Supporting information for:

\title{
A metabolism-coupled cell-independent acetylcholinesterase activity assay for evaluation of the effects of chlorination on diazinon toxicity
}

\author{
Taku Matsushita ${ }^{*}$, Yuji Kikkawa $\$$ Kei Omori ${ }^{\ddagger}$ \\ Yoshihiko Matsui†, and Nobutaka Shirasaki ${ }^{\dagger}$ \\ †Faculty of Engineering, Hokkaido University, N13W8, Sapporo 060-8628, Japan \\ *Graduate School of Engineering, Hokkaido University, N13W8, Sapporo 060-8628, Japan \\ ${ }^{*}$ Corresponding author: taku-m@eng.hokudai.ac.jp; +81-11-706-7279
}

\section{Contents}

$\checkmark \quad$ Fig. S1 Chemical structures of diazinon and diazinon-oxon. $\quad$ S3

$\checkmark \quad$ Table S1 Composition of stock solution of S9 mix. $\quad$ S3

$\checkmark \quad$ Text S1 Quantification of choline, diazinon, and diazinon-oxon. $\quad$ S4

$\checkmark \quad$ Fig. S2 Concentrations of DOC derived from diazinon during chlorination and the structures of three of its transformation products.

$\checkmark \quad$ Fig. S3 Concentrations of DOC derived from diazinon during chlorination after metabolism.S5

$\checkmark \quad$ Fig. S4 Indirect AChE activity induced by diazinon and diazinon-oxon.

$\checkmark \quad$ Fig. S5 Schematic diagram of possible transformations of diazinon during chlorination and metabolism.

$\checkmark \quad$ Fig. S6 Comparison of LC chromatograms of diazinon, diazinon-oxon, and control subjected to acetylcholinesterase activity assay after metabolism.

$\checkmark \quad$ Fig. S7 Changes in the area of Peak 2 on the LC chromatograms during chlorination after metabolism.

$\checkmark \quad$ Table S2 Candidate molecular formulae for the compound represented by Peak $2 . \quad$ S8

$\checkmark \quad$ Fig. S8 Candidate chemical structures of the metabolite detected as Peak 2. S9

$\checkmark \quad$ Fig. S9 Tandem mass spectrometry spectra of Peak 2 with different values of collision energy and assignment of fragment ions for Structure S8a.

$\checkmark \quad$ Fig. S10 Tandem mass spectrometry spectra of Peak 2 with different values of collision energy and assignment of fragment ions for Structure $S 8 b$.

$\checkmark \quad$ Fig. S11 Tandem mass spectrometry spectra of Peak 2 with different values of collision energy and assignment of fragment ions for Structure S8c.

$\checkmark \quad$ Fig. S12 Tandem mass spectrometry spectra of Peak 2 with different values of collision energy and assignment of fragment ions for Structure $\mathrm{S} 8 \mathrm{~d}$.

$\checkmark \quad$ Text S2 Results and interpretation of in silico site-of-metabolism analyses. 
$\checkmark \quad$ Table S3 Comparison of probabilities obtained using FAME 2 for the identified candidate structures of the metabolite with anti-AChE activity.

$\checkmark \quad$ Table S4 Comparison of ranks obtained using RS-WebPredictor for the identified candidate structures of the metabolite with anti-AChE activity.

$\checkmark$ Table S5 Comparison of scores obtained using SMARTCyp for the identified candidate structures of the metabolite with anti-AChE activity.

$\checkmark \quad$ Table S6 Comparison of delta P values obtained using SOMP for the identified candidate structures of the metabolite with anti-AChE activity. 
(a) Diazinon<smiles>CCOP(=S)(OCC)Oc1cc(C)nc(C(C)C)n1</smiles>

(b) Diazinon-oxon<smiles>CCOP(=O)(OCC)Oc1cc(C)nc(C(C)C)n1</smiles>

Fig. S1 Chemical structures of (a) diazinon and (b) diazinon-oxon.

Table S1 Composition of stock solution of S9 mix.

\begin{tabular}{lr}
\hline Component & Amount \\
\hline \hline S9 & $2 \mathrm{~mL}$ \\
glucose-6-phosphate & $37.6 \mathrm{mg}$ \\
$\beta$-NADPH & $70.8 \mathrm{mg}$ \\
$\beta$-NADH & $53.2 \mathrm{mg}$ \\
$0.4 \mathrm{M} \mathrm{MgCl}{ }_{2}$ solution & $400 \mu \mathrm{L}$ \\
1.65 M KCl solution & $400 \mu \mathrm{L}$ \\
$0.2 \mathrm{M}$ phosphate buffer * & $10 \mathrm{~mL}$ \\
Milli-Q water & $8 \mathrm{~mL}$ \\
\hline & \\
& \\
\hline * Phosphate buffer & $\mathrm{Amount}$ \\
\hline \hline $\mathrm{NaH}_{2} \mathrm{PO}_{4} \cdot 2 \mathrm{H}_{2} \mathrm{O}$ & $15.7 \mathrm{~g}$ \\
$\mathrm{Na}_{2} \mathrm{HPO}_{4} \cdot 12 \mathrm{H}_{2} \mathrm{O}$ & $35.9 \mathrm{~g}$ \\
$\mathrm{Milli}^{-Q}$ water & $500 \mathrm{~mL}$ \\
\hline
\end{tabular}




\section{Text S1 Quantification of choline, diazinon, and diazinon-oxon}

The concentration of choline was measured by using a hybrid quadrupole-orbitrap mass spectrometer (MS; Q-Exactive, Thermo Fisher Scientific Inc., Waltham, MA, USA) coupled with a liquid chromatograph (LC; UltiMate 3000, Thermo Fisher Scientific). A 5- $\mu \mathrm{L}$ sample of the test solution was assayed with the LC equipped with a $100 \mathrm{~mm} \times 2.1 \mathrm{~mm}$ Cortecs UPLC HILIC column $(1.6-\mu \mathrm{m}$ particle size, Waters Corporation, Milford, MA, USA). The mobile phase was a binary gradient of $100 \mathrm{mM}$ ammonium formate in Milli-Q water (solvent A) and 100\% acetonitrile (solvent B) at a flow rate of $200 \mu \mathrm{L} / \mathrm{min}$ as follows: begin with 95:5 (v/v) A/B, increase linearly to $60 \%$ B over a period of $0.75 \mathrm{~min}$, hold at that ratio for $0.25 \mathrm{~min}$, decrease linearly to $30 \% \mathrm{~B}$ over a period of $0.25 \mathrm{~min}$, hold at that ratio for $1.25 \mathrm{~min}$, and then decrease linearly to $5 \% \mathrm{~B}$ over a period of $3.5 \mathrm{~min}$. The MS was operated in electrospray ionization mode (positive) with a spray voltage of $3.2 \mathrm{kV}$. The temperature of the capillary and electrospray ionization probe heater was 220 and $450{ }^{\circ} \mathrm{C}$, respectively. The flow rate of the sheath gas, auxiliary gas, and sweep gas was 50, 15, and 0 units, respectively. The S-lens radio frequency level was set to 57 . The concentration of choline was quantified in selected-ionmonitoring mode with $\mathrm{m} / \mathrm{z} 104.1070$ (resolution $=70,000$ ). The detection limit for choline was $5 \mathrm{nM}$.

The concentrations of diazinon and diazinon-oxon were measured with the same apparatus as used for choline. A 5- $\mu \mathrm{L}$ sample of test solution was assayed with the $\mathrm{LC}$ equipped with a $50 \mathrm{~mm} \times 2.1$ mm Hypersil Gold column (1.9- $\mu \mathrm{m}$ particle size, Thermo Fisher Scientific). The mobile phase was a binary gradient of $2 \mathrm{mM}$ ammonium formate in Milli-Q water (solvent A) and 100\% methanol (solvent B) at a flow rate of $200 \mu \mathrm{L} / \mathrm{min}$ as follows: 99:1 (v/v) A/B for $2 \mathrm{~min}$, increase linearly to $60 \% \mathrm{~B}$ over a period of $2 \mathrm{~min}$, increase linearly to $75 \% \mathrm{~B}$ over a period of $5 \mathrm{~min}$, increase linearly to $99 \%$ B over a period of $5 \mathrm{~min}$, decrease linearly to $1 \% \mathrm{~B}$ over a period of $0.1 \mathrm{~min}$, and then hold at that ratio for $1.9 \mathrm{~min}$. The MS was operated in electrospray ionization mode (positive) with a spray voltage of $3.2 \mathrm{kV}$. The temperature of the capillary and electrospray ionization probe heater was 220 and $450{ }^{\circ} \mathrm{C}$, respectively. The flow rate of the sheath gas, auxiliary gas, and sweep gas was 50, 15, and 0 units, respectively. The S-lens radio frequency level was set to 57 . The concentrations of diazinon and diazinon-oxon were quantified in selected-ion-monitoring mode with $\mathrm{m} / z 305.1083$ and 289.1312 , respectively (resolution $=70,000$ ). The detection limit was $3 \mathrm{nM}$ both for diazinon and diazinon-oxon. 


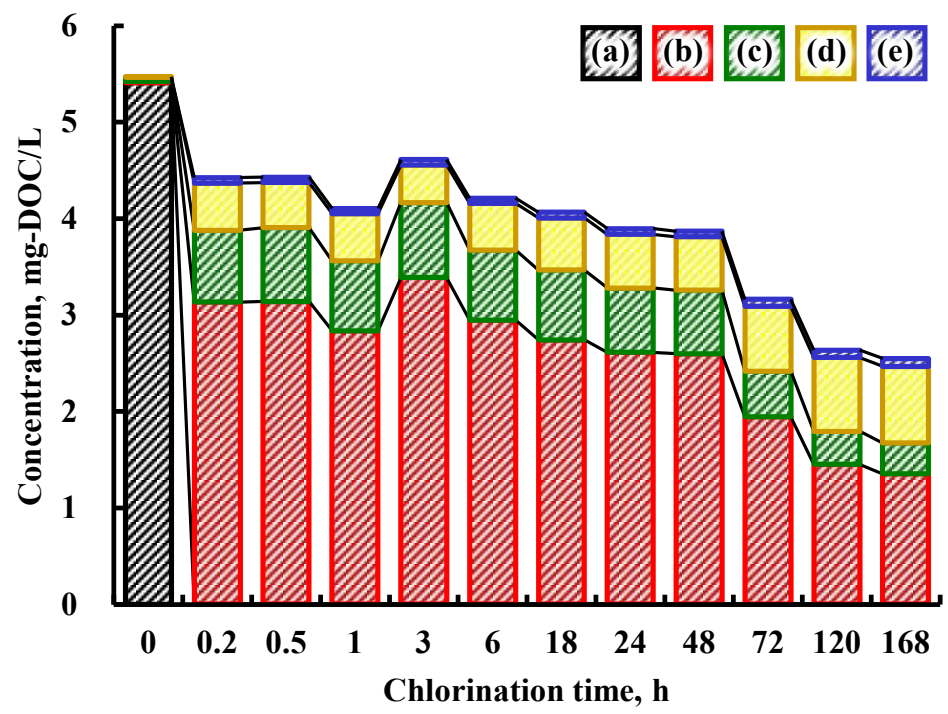<smiles>Cc1cc(O)nc(C(C)C)n1</smiles>

(c) 2-isopropyl-6-methyl-pyrimidin-4-ol (IMP)<smiles>CCOP(=O)(O)OCC</smiles>

(d) diethyl phosphate<smiles>COP(=O)(O)OC</smiles>

(e) dimethyl phosphate

Fig. S2 Concentrations of dissolved organic carbon (DOC) derived from diazinon during chlorination and the structures of three of its transformation products. (a) Diazinon, (b) diazinon-oxon, (c) 2-isopropyl-6-methyl-pyrimidin-4-ol (IMP), (d) diethyl phosphate, and (e) dimethyl phosphate.

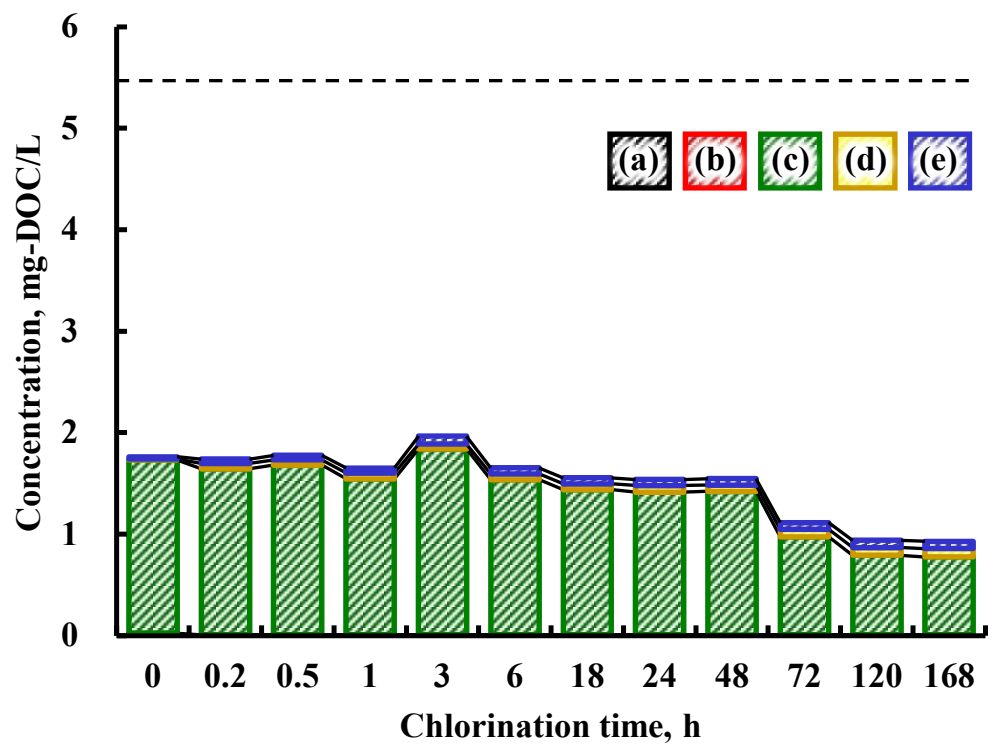

Fig. S3 Concentrations of dissolved organic carbon (DOC) derived from diazinon during chlorination after metabolism. (a) Diazinon, (b) diazinon-oxon, (c) 2-isopropyl-6-methyl-pyrimidin-4-ol (IMP), (d) diethyl phosphate, and (e) dimethyl phosphate. Dashed line indicates the DOC concentration of the initial sample (before chlorination) before metabolism. 


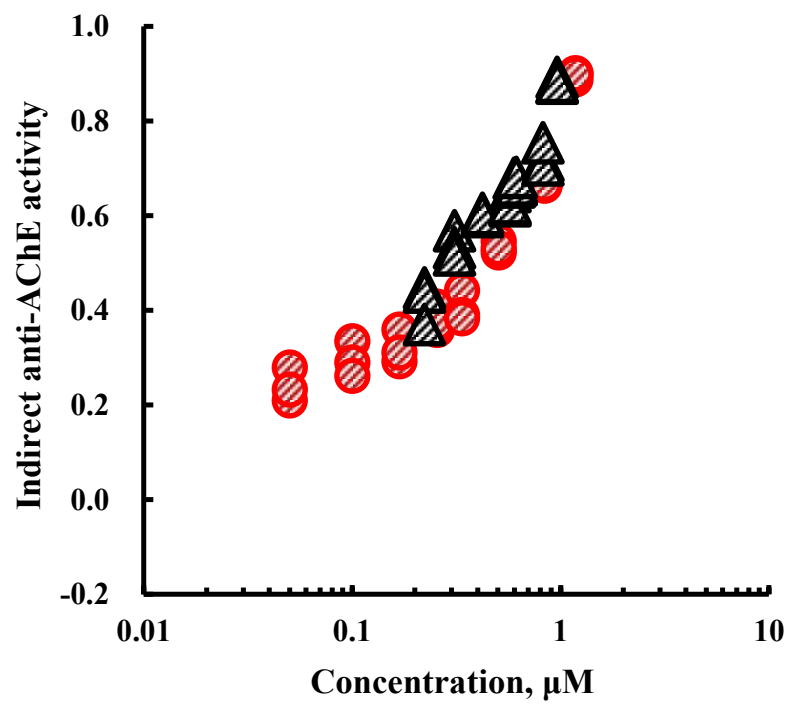

Fig. S4 Indirect anti-acetylcholinesterase (AChE) activity induced by diazinon (black triangles) and diazinon-oxon (red circles). The values are the same as those in Fig. 1. Note that the concentrations are before metabolism.

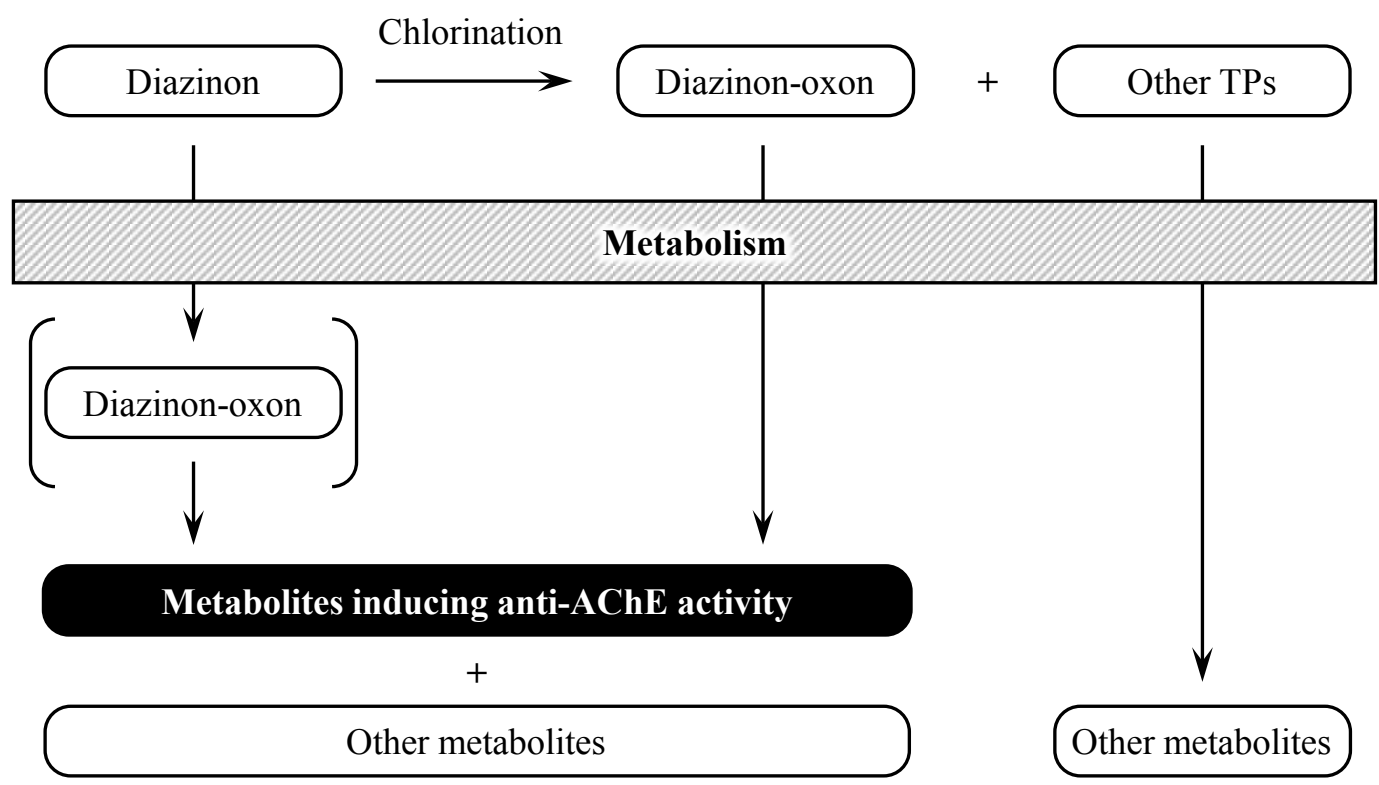

Fig. S5 Schematic diagram of possible transformations of diazinon during chlorination and metabolism. AChE, acetylcholinesterase; TPs, transformation products. 
(a) Diazinon

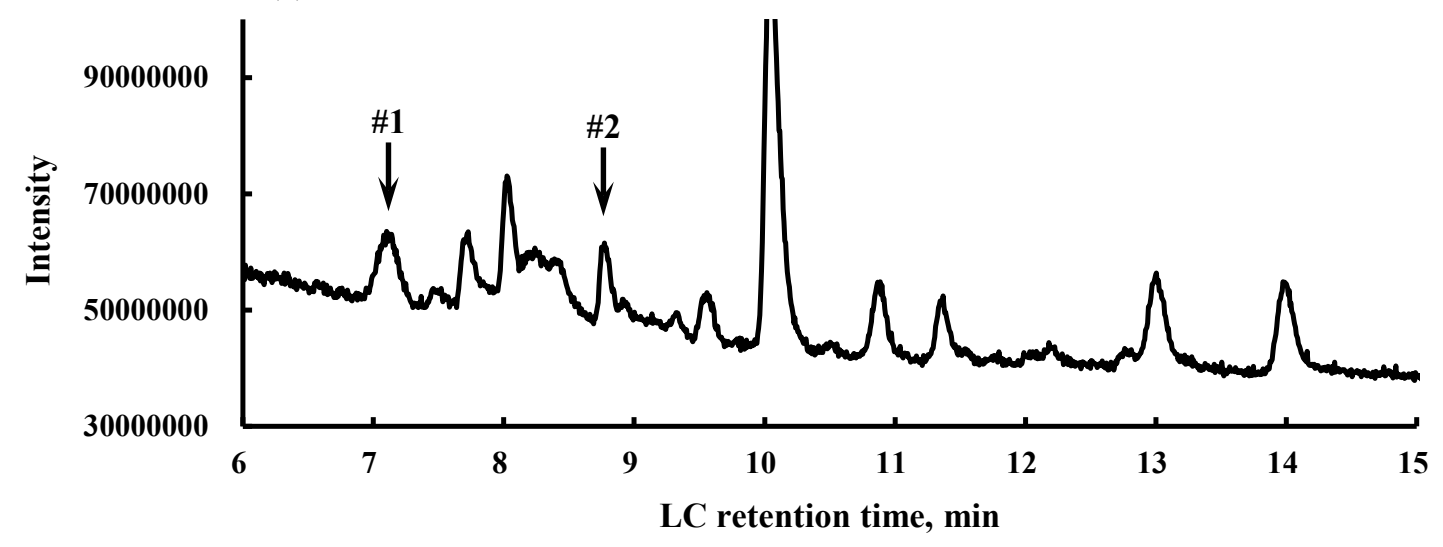

(b) Diazinon-oxon

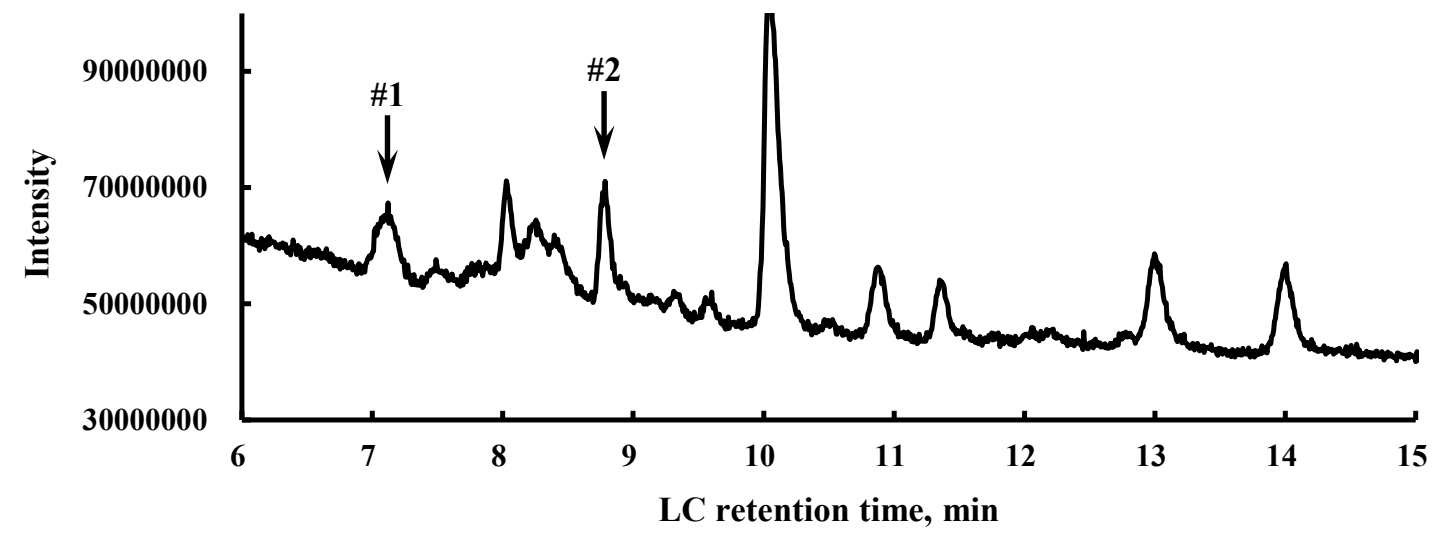

(c) Control

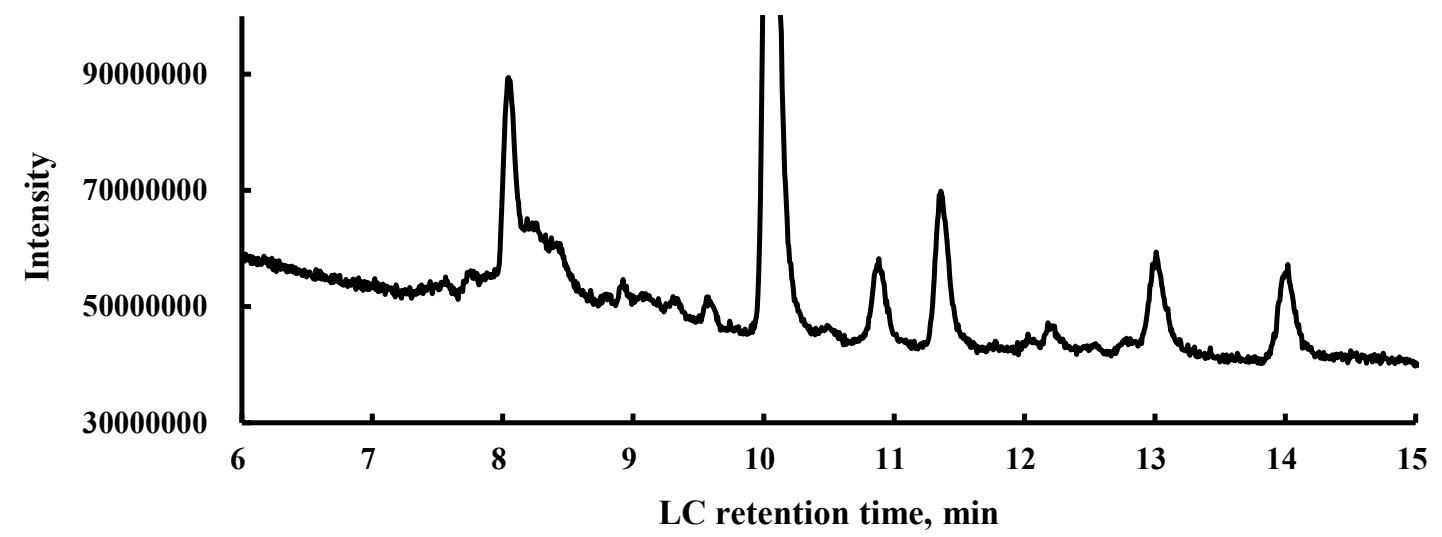

Fig. S6 Comparison of liquid chromatography (LC) chromatograms (positive ion mode) of (a) diazinon, (b) diazinon-oxon, and (c) control subjected to acetylcholinesterase activity assay after metabolism. The control sample was prepared as follows: $800 \mu \mathrm{L}$ phosphate buffer was mixed with $2000 \mu \mathrm{L}$ of the stock solution of S9 mix, and then incubated at $37^{\circ} \mathrm{C}$ for $20 \mathrm{~min}$ in a shaking water bath. After that, $900 \mu \mathrm{L}$ of the solution was immediately centrifuged for 60 min by using an ultracentrifuge. 


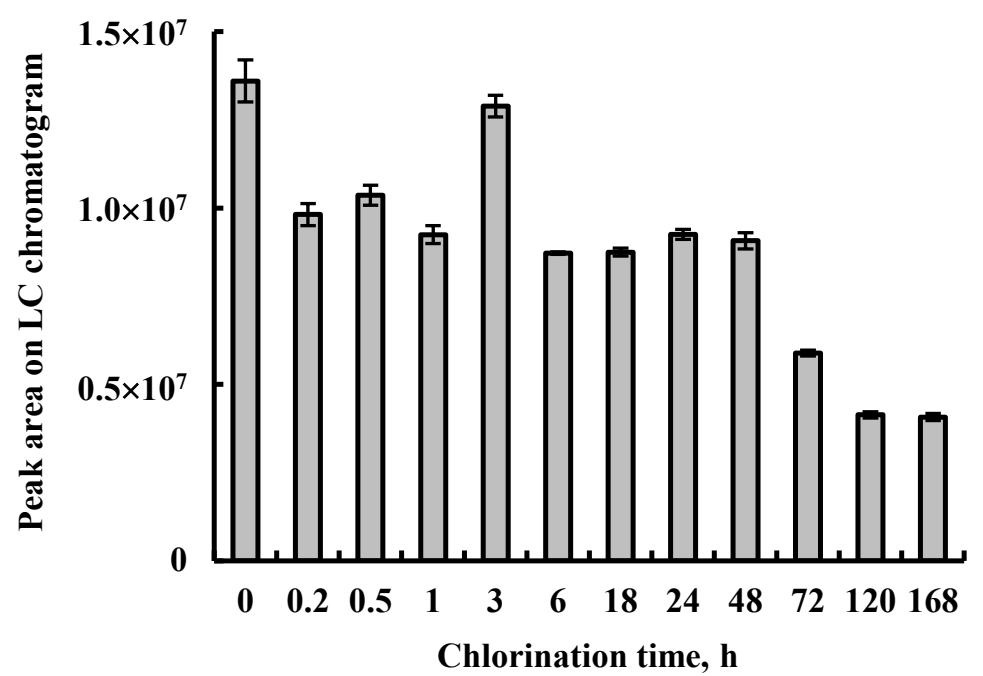

Fig. S7 Changes in the area of Peak 2 on the liquid chromatography chromatograms (positive ion mode) during chlorination after metabolism. Error bars indicate standard deviations of 3 measurements.

Table S2 Candidate molecular formulae for the compound represented by Peak $2(\mathrm{~m} / \mathrm{z}=305.1256)$.

\begin{tabular}{lcc}
\hline Molecular formula & Theoretical $\mathrm{m} / z$ & Error, ppm \\
\hline \hline $\mathrm{C}_{12} \mathrm{H}_{22} \mathrm{O}_{5} \mathrm{~N}_{2} \mathrm{P}$ & 305.1261 & -1.6 \\
$\mathrm{C}_{9} \mathrm{H}_{24} \mathrm{O}_{8} \mathrm{NP}$ & 305.1234 & 7.2 \\
$\mathrm{C}_{13} \mathrm{H}_{21} \mathrm{O}_{8}$ & 305.1231 & 8.2 \\
\hline
\end{tabular}

Calculation condition: $\mathrm{C} \leq 14, \mathrm{H} \leq 50, \mathrm{O} \leq 10, \mathrm{~N} \leq 2, \mathrm{P} \leq 1$, and $\mathrm{S} \leq 1$. 

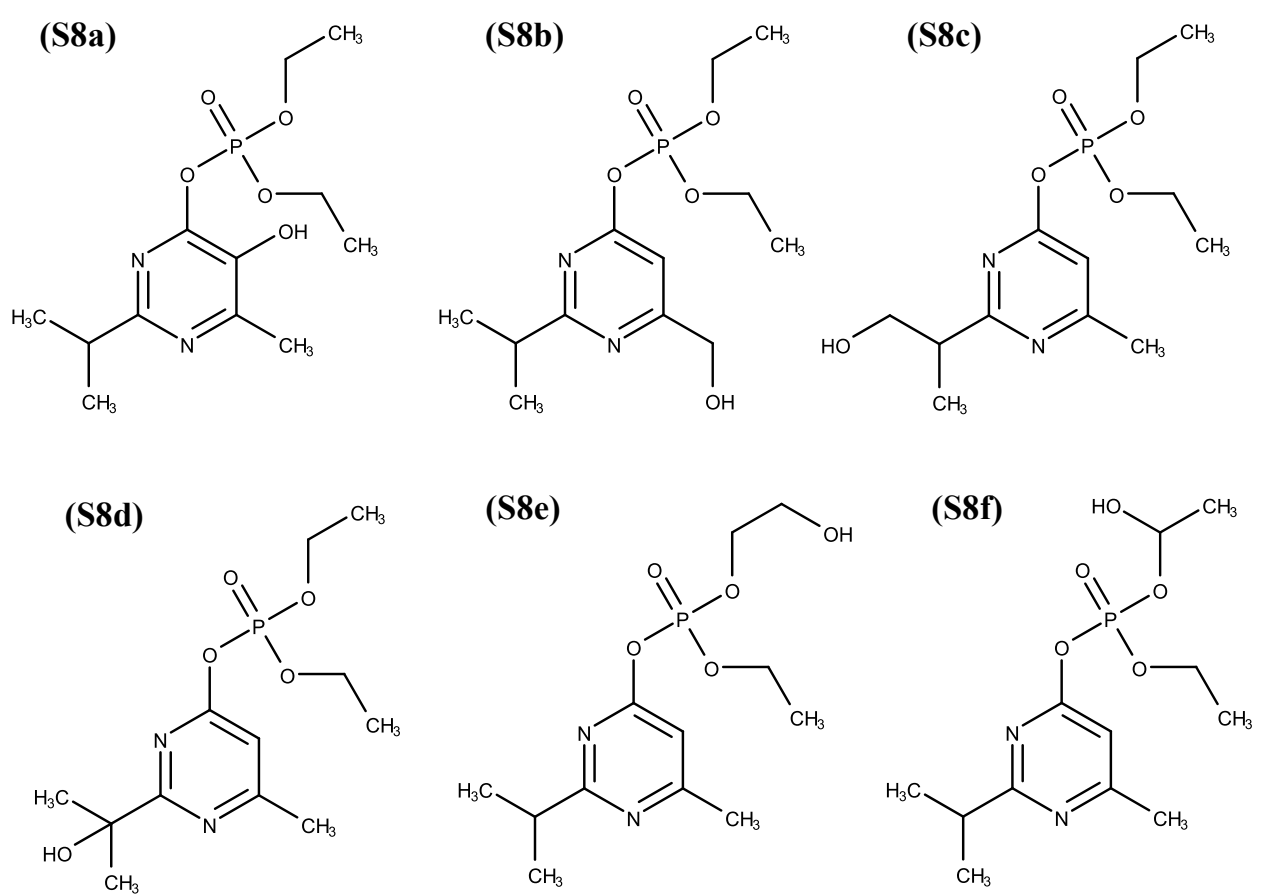

Fig. S8 Candidate chemical structures of the metabolite detected as Peak 2. 
(a) $\mathrm{CE}=10 \mathrm{eV}$, annotation for Structure S8a

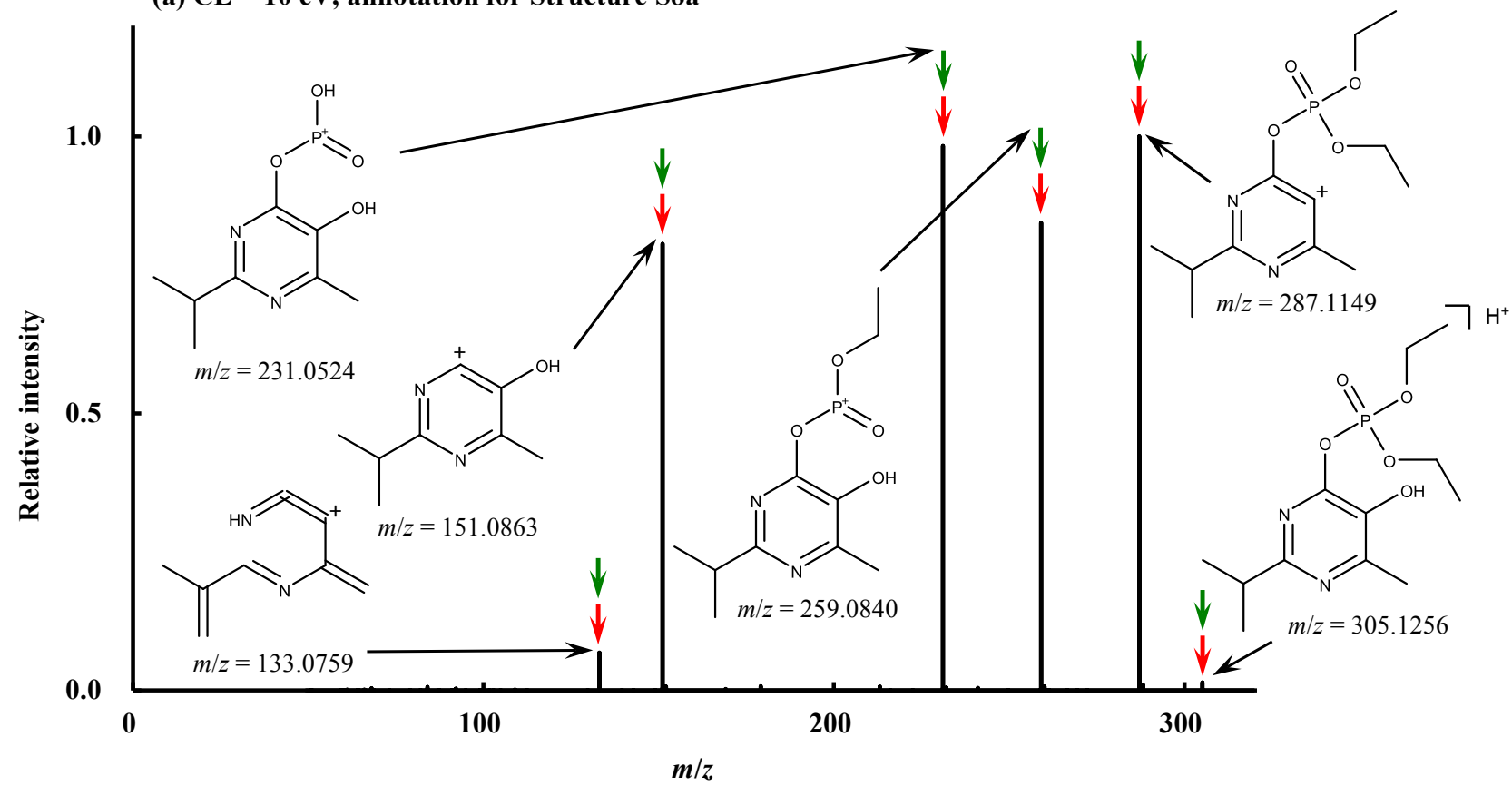

(b) $\mathrm{CE}=\mathbf{5 0} \mathrm{eV}$, annotation for Structure S8a

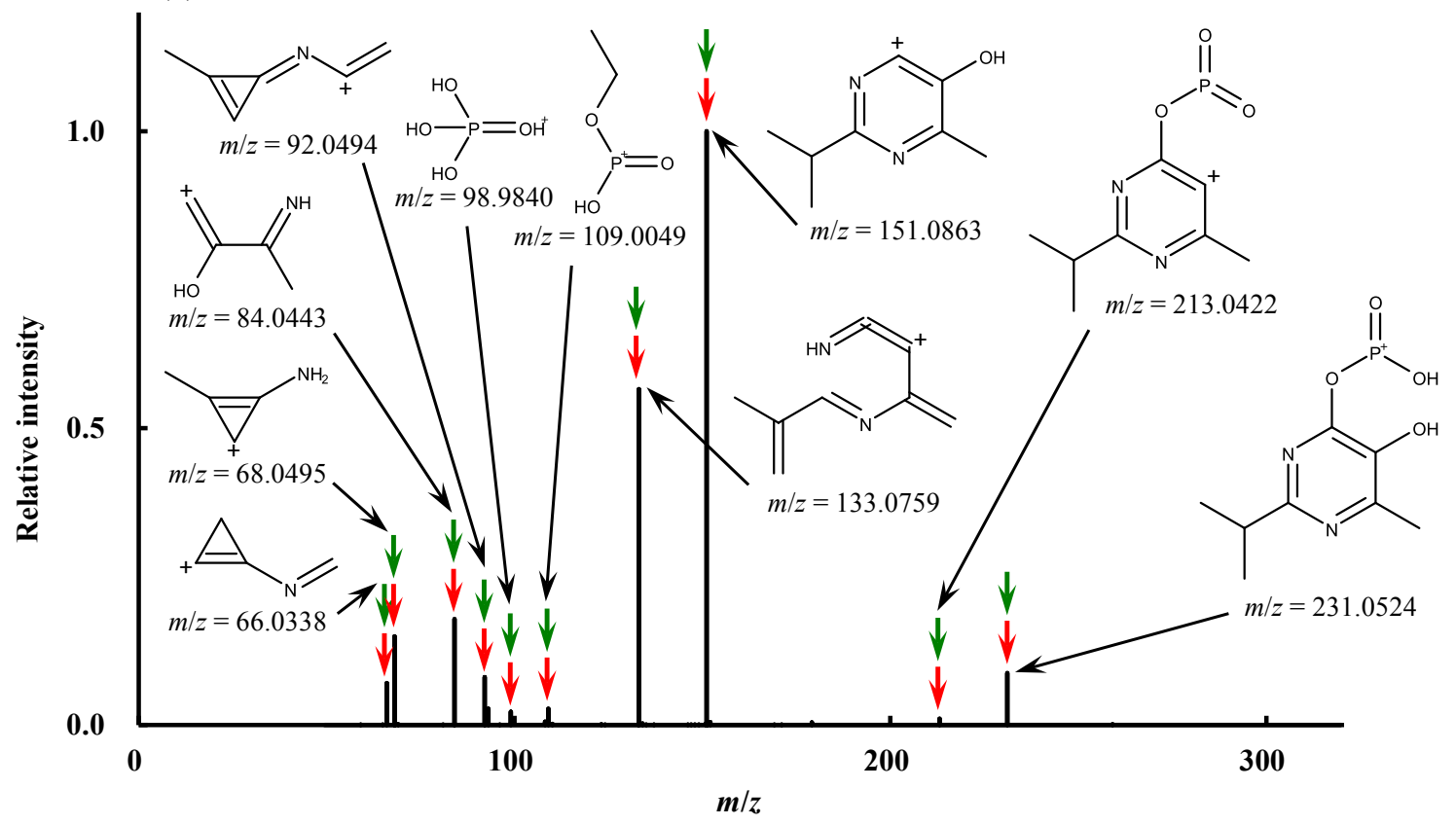

Fig. S9 Tandem mass spectrometry spectra of Peak $2(\mathrm{~m} / \mathrm{z}=305.1256$, positive ion mode) with different values of collision energy (CE) and assignment of fragment ions for Structure S8a. Red and green arrows represent fragment ions assigned with Mass Frontier and MAGMa, respectively. 
(a) $\mathrm{CE}=10 \mathrm{eV}$, annotation for Structure $\mathrm{S} 8 \mathrm{~b}$

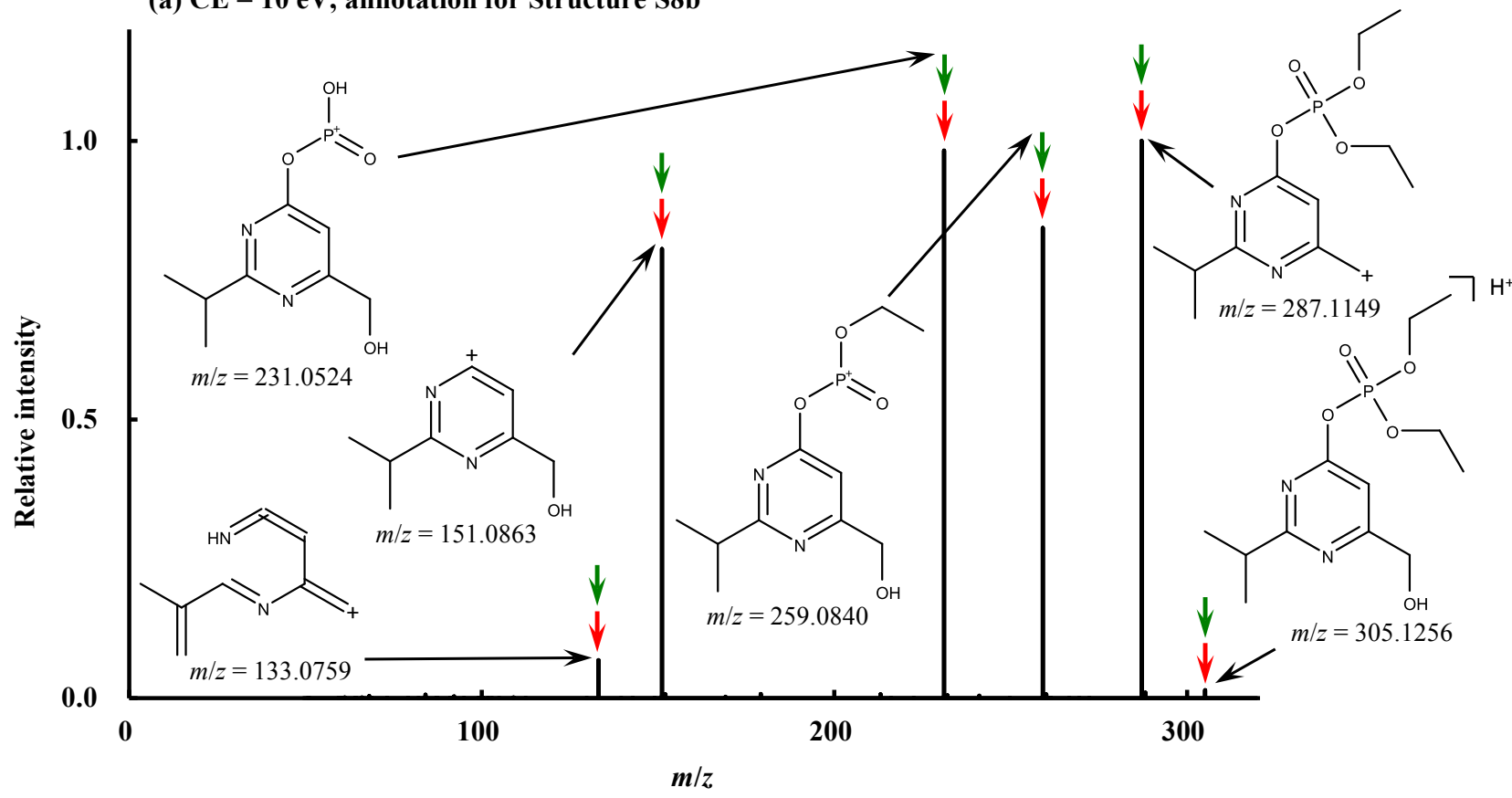

(b) $\mathrm{CE}=50 \mathrm{eV}$, annotation for Structure $\mathbf{S 8 b}$

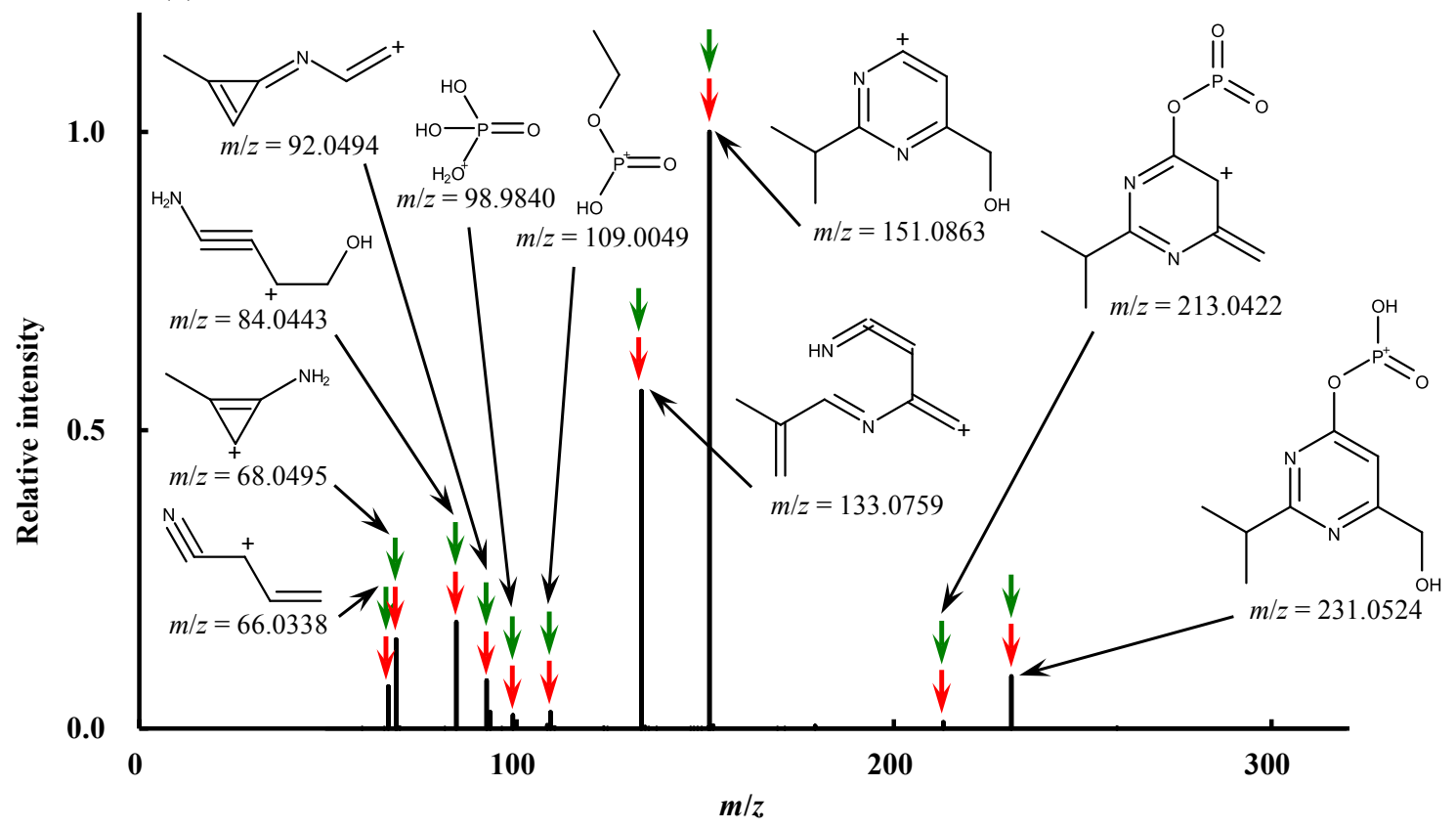

Fig. S10 Tandem mass spectrometry spectra of Peak $2(\mathrm{~m} / \mathrm{z}=305.1256$, positive ion mode) with different values of collision energy (CE) and assignment of fragment ions for Structure S8b. Red and green arrows represent fragment ions assigned with Mass Frontier and MAGMa, respectively. 
(a) $\mathrm{CE}=10 \mathrm{eV}$, annotation for Structure S8c

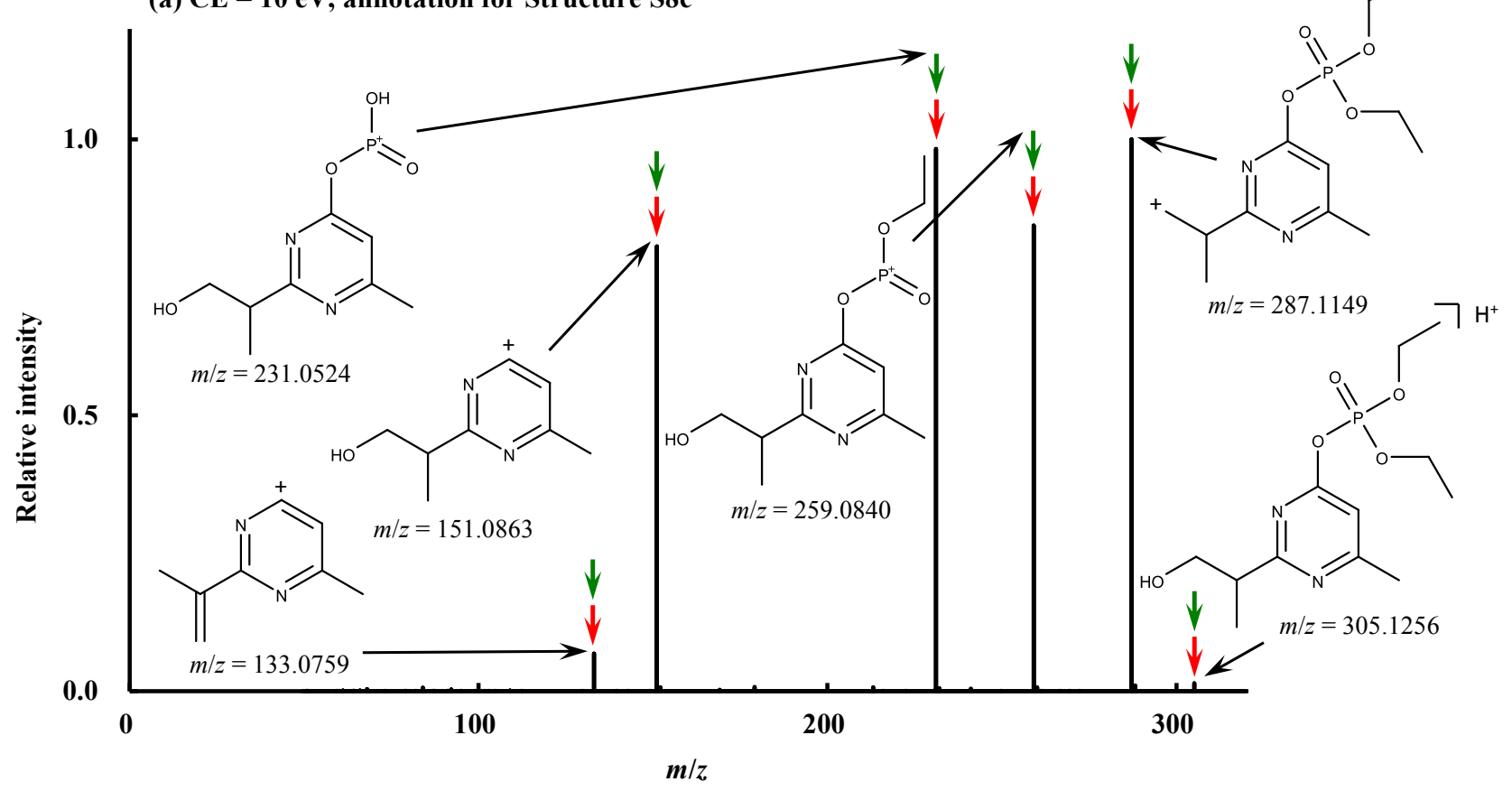

(b) $\mathrm{CE}=\mathbf{5 0} \mathrm{eV}$, annotation for Structure S8c

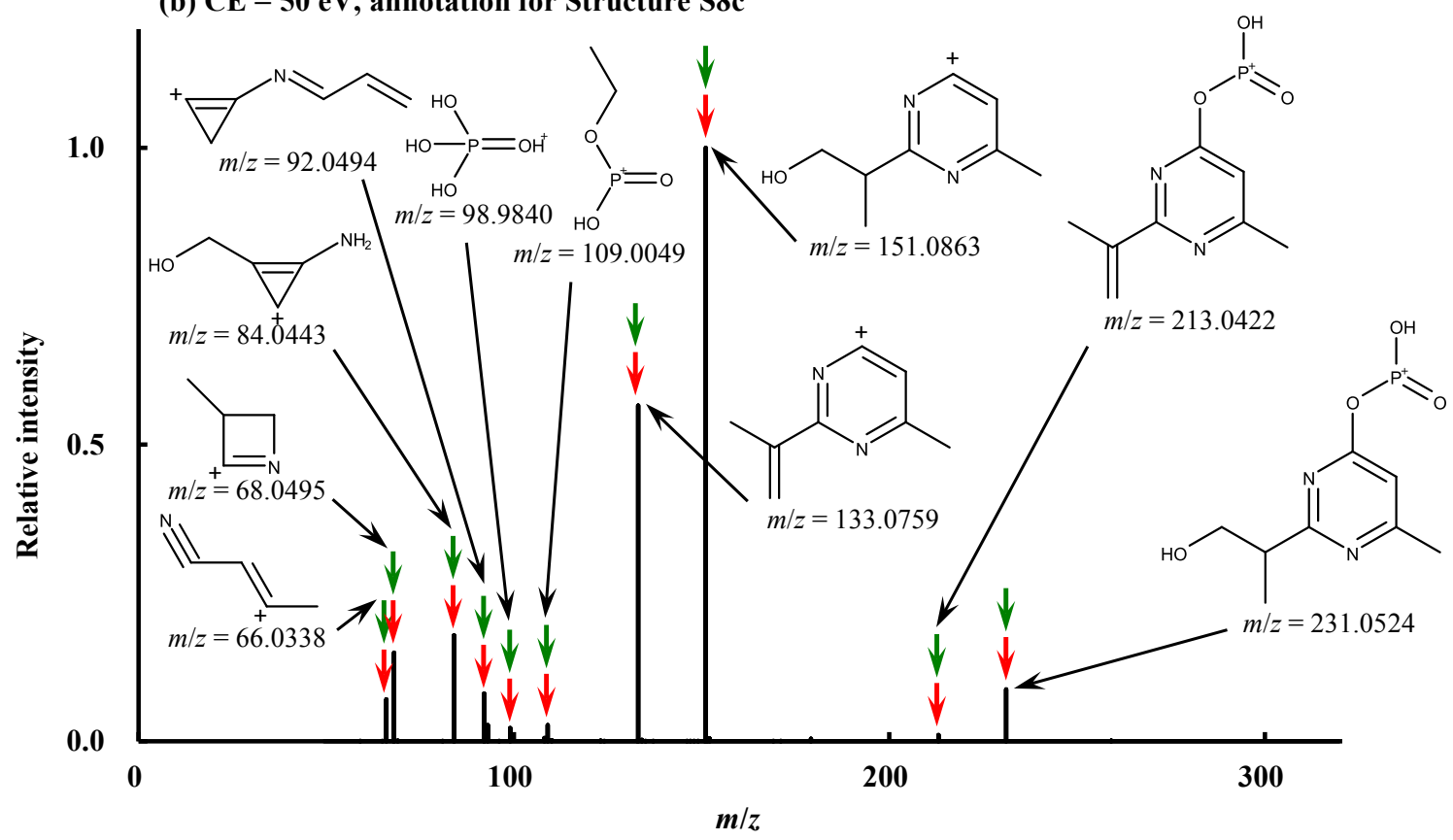

Fig. S11 Tandem mass spectrometry spectra of Peak $2(\mathrm{~m} / \mathrm{z}=305.1256$, positive ion mode) with different values of collision energy (CE) and assignment of fragment ions for Structure S8c. Red and green arrows represent fragment ions assigned with Mass Frontier and MAGMa, respectively. 
(a) $\mathrm{CE}=10 \mathrm{eV}$, annotation for Structure S8d

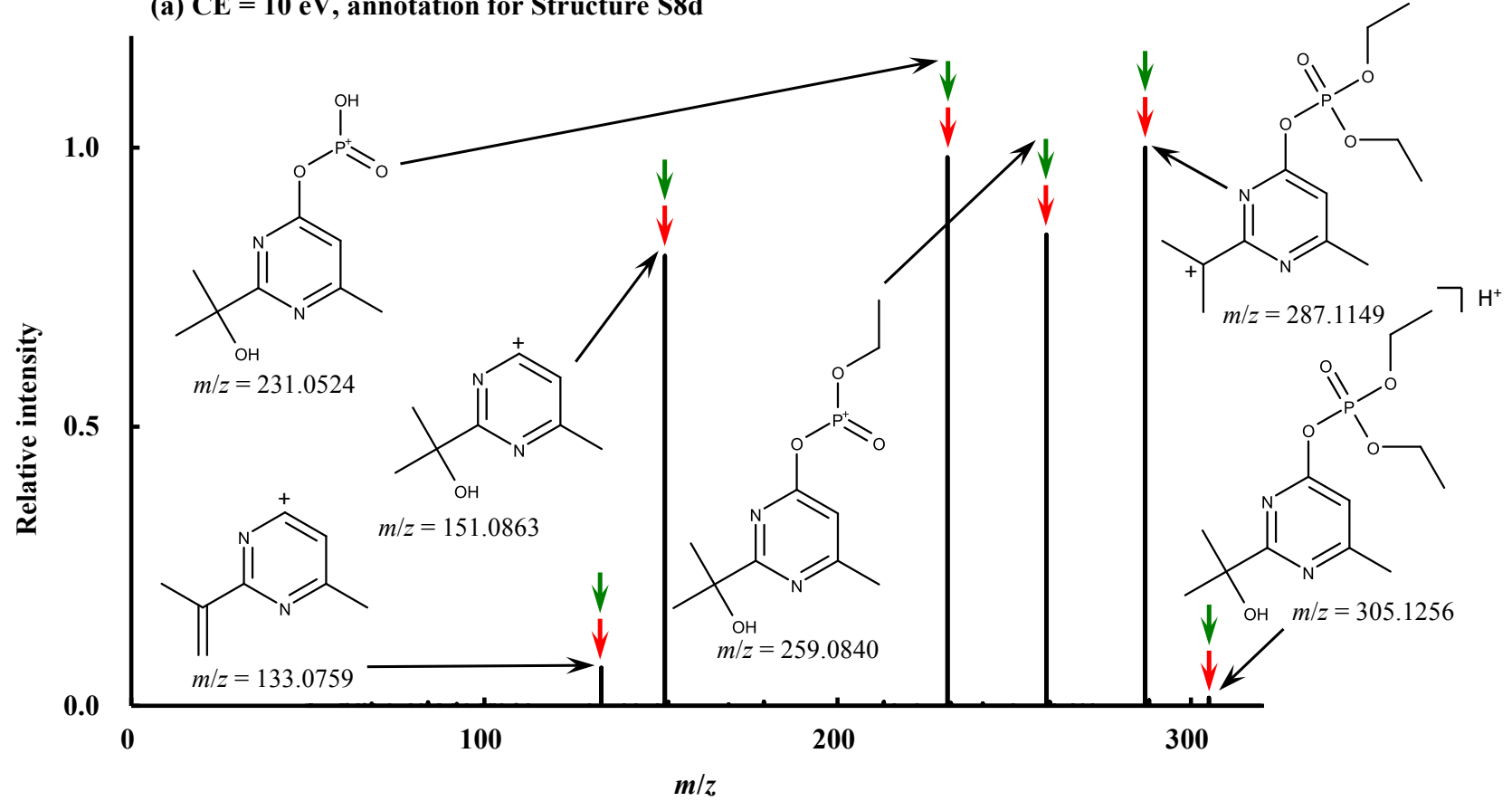

(b) $\mathrm{CE}=\mathbf{5 0} \mathrm{eV}$, annotation for Structure S8d

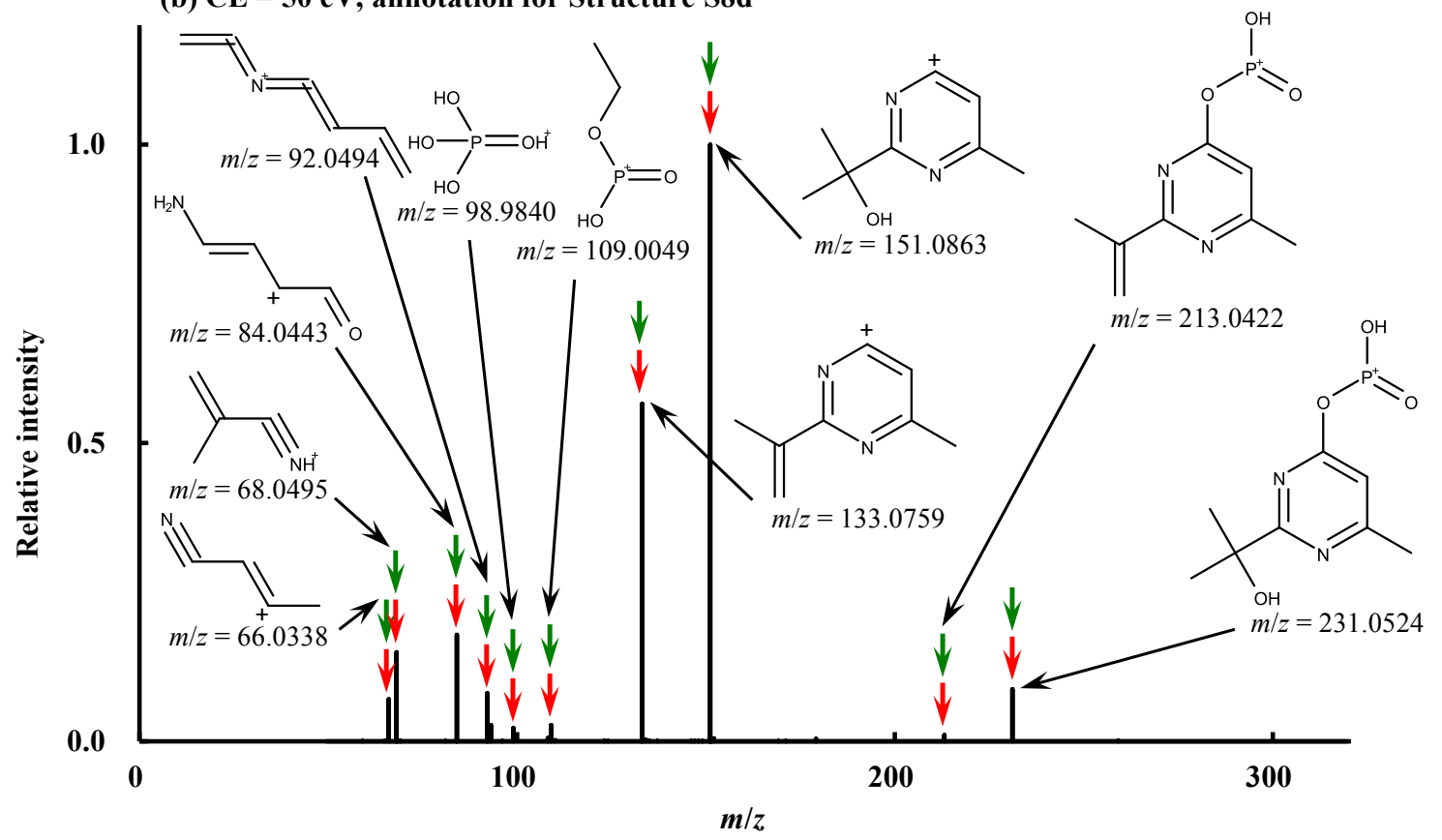

Fig. S12 Tandem mass spectrometry spectra of Peak $2(\mathrm{~m} / \mathrm{z}=305.1256$, positive ion mode) with different values of collision energy (CE) and assignment of fragment ions for Structure S8d. Red and green arrows represent fragment ions assigned with Mass Frontier and MAGMa, respectively. 


\section{Text S2 Results and interpretation of in silico site-of-metabolism analyses}

FAst MEtabolizer 2 (FAME 2) ${ }^{1}$ outputs for the four identified metabolite candidate structures (Table S3). Probabilities were generated for each atom in the structure by using the extra trees model (i.e., the percentage of trees that predicted the atom as a site of metabolism [SOM]); the higher the probability is, the more likely the structure is enzymatically produced by CYP. Structures S8a and S8d showed the largest probabilities, followed by S8b and S8c in that order.

Table S3 Comparison of probabilities obtained using FAME 2 for the identified candidate structures of the metabolite with anti-acetylcholinesterase activity. The higher the probability is, the smaller the rank is.

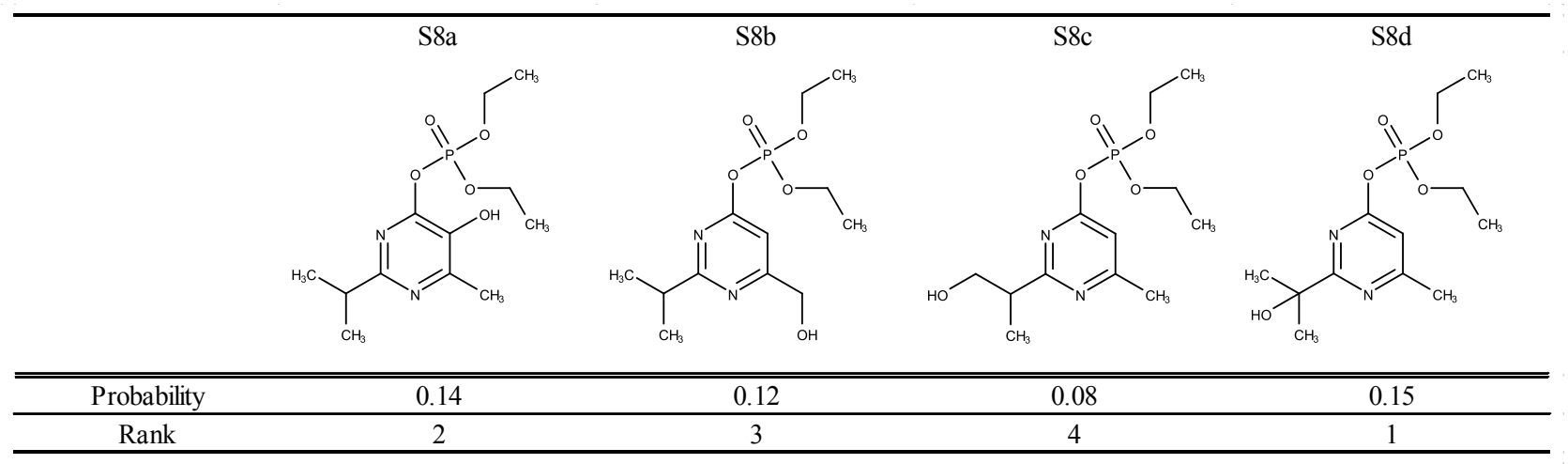

RS-WebPredictor ${ }^{2}$ outputs for the top three predicted SOMs for each CYP isozyme for each candidate structure (Table S4). Structures not in the top 3 were assigned rank 4. S8a and S8b were ranked within the top 3 for almost all isozymes, whereas S8c and S8d were ranked below the top 3 for almost all isozymes. The candidate structures were ordered based on their average ranks.

Table S4 Comparison of ranks obtained using RS-WebPredictor for the identified candidate structures of the metabolite with anti-acetylcholinesterase activity.
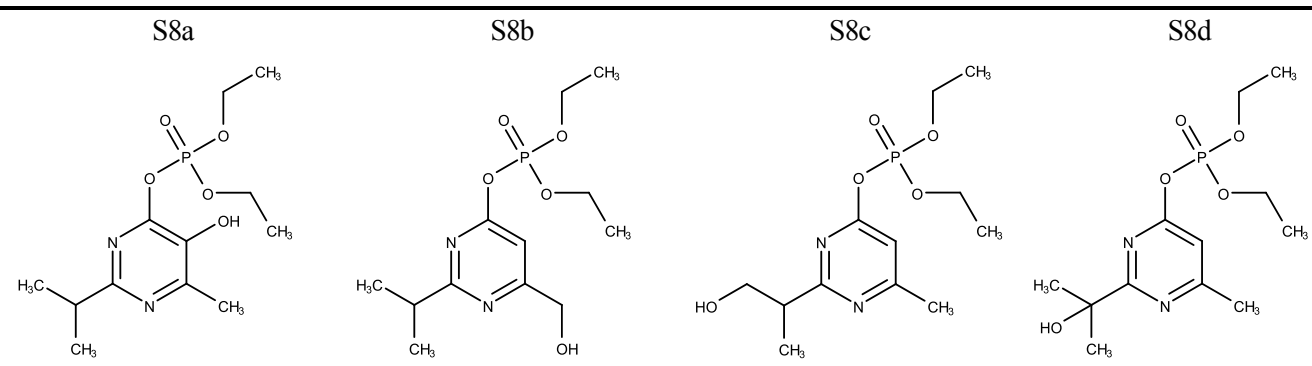

\begin{tabular}{ccccc}
\hline \hline CYP1A2 & 3 & 2 & 4 & 4 \\
CYP2A6 & 3 & 1 & 4 & 4 \\
CYP2B6 & 2 & 1 & 4 & 4 \\
CYP2C8 & 2 & 1 & 4 & 4 \\
CYP2C9 & 2 & 1 & 4 & 4 \\
CYP2C19 & 3 & 1 & 4 & 4 \\
CYP2D6 & 4 & 1 & 4 & 4 \\
CYP2E1 & 4 & 1 & 4 & 2 \\
CYP3A4 & 3 & 1 & 4 & 4 \\
\hline \hline Average & 2.9 & 1.1 & 4.0 & 3.8 \\
\hline Average rank & 2 & 1 & 4 & 3 \\
\hline
\end{tabular}


SMARTCyp $^{3}$ outputs for the candidate structures (Table S5). A score was calculated by using the reactivity descriptor (an estimate of the energy required for the CYP under consideration to react with a given atom in the molecule) and the accessibility descriptor (the longest bond-path distance from the atom under consideration divided by the longest bond-path distance across the whole molecule). The atoms in the molecule were ranked by score, with the lowest score resulting in the lowest rank, and thus the highest probability of being a SOM. The scores for S8b and S8d were comparable for all CYP isozymes, and were always lower than those for S8a and S8c.

Table S5 Comparison of scores obtained using SMARTCyp for the identified candidate structures of the metabolite with anti-acetylcholinesterase activity. The lower the score is, the smaller the rank is.

\begin{tabular}{cccccccccc}
\hline \\
Score
\end{tabular}

$\mathrm{SOMP}^{4}$ outputs for the candidate structures (Table S6). Delta $\mathrm{P}$ values were calculated by subtracting the probability that a given atom is not a SOM for the CYP isozyme under consideration from the probability that the atom is a SOM. Accordingly, the higher the delta $\mathrm{P}$ value is, the more likely the structure is enzymatically produced by the CYP. The delta P values of S8b and S8d were positive for all CYP isozymes, whereas those of S8a and S8c were negative for more than half of the CYP isozymes and were always smaller than those of S8b and S8d.

Table S6 Comparison of delta P values obtained using SOMP for the identified candidate structures of the metabolite with anti-acetylcholinesterase activity. The higher the delta $\mathrm{P}$ value is, the smaller the rank is.

\begin{tabular}{|c|c|c|c|c|c|}
\hline & & S8a & $\mathrm{S} 8 \mathrm{~b}$ & S8c & S8d \\
\hline \multirow{6}{*}{ Delta P } & CYP1A2 & 0.37 & 0.57 & -0.20 & 0.88 \\
\hline & CYP2C19 & -0.25 & 0.58 & 0.33 & 0.27 \\
\hline & CYP2C9 & -0.29 & 0.65 & -0.07 & 0.91 \\
\hline & CYP2D6 & -0.17 & 0.17 & -0.53 & 0.92 \\
\hline & CYP3A4 & 0.41 & 0.53 & 0.04 & 0.89 \\
\hline & Average & 0.01 & 0.50 & -0.09 & 0.77 \\
\hline \multicolumn{2}{|c|}{ Average rank } & 3 & 2 & 4 & 1 \\
\hline
\end{tabular}




\section{References}

(1) Šícho, M., de Bruyn Kops, C., Stork, C., Svozil, D. and Kirchmair, J. (2017) FAME 2: simple and effective machine learning model of cytochrome P450 regioselectivity, Journal of Chemical Information and Modeling, 57, 1832-1846.

(2) Zaretzki, J., Bergeron, C., Huang, T., Rydberg, P., Swamidass, S. J. and Breneman, C. M. (2013) RSWebPredictor: a server for predicting CYP-mediated sites of metabolism on drug-like molecules, Bioinformatics, 29, 497-498.

(3) Rydberg, P., Gloriam, G. E., Zaretzki, J., Breneman, C. and Olsen, L. (2010) SMARTCyp: a 2D method for prediction of cytochrome P450-mediated drug metabolism, ACS Medicinal Chemistry Letters, 1, 96-100.

(4) http://www.way2drug.com/SOMP/interpr.php, visited on 29 June 2021. 